\title{
ESTABILIDADE SOBRE A ÓTICA ESTATÍSTICA.
}

\author{
A. C. NASCIMENTO ${ }^{1}$, J. MANZI $^{2}$ \\ ${ }^{1,2}$ Universidade Federal de Campina Grande, Departamento de Engenharia Química \\ Alisson_Nascimento@yahoo.com,br
}

\begin{abstract}
RESUMO - Este trabalho apresenta uma metodologia baseada em princípios da estatística clássica para determinar as regiões de estabilidade robusta de sistemas lineares ou não lineares, com um nível de conservadorismo muito menor que àqueles apresentados pelas metodologias determinísticas. O caráter aleatório das raízes do sistema, representado pelas distribuições de probabilidades diz respeito ao universo de todos os resultados possíveis para o fenômeno, garantindo o mapeamento através da métrica de distância estatística associada a função chi-quadrado para um aceitável o nível do erro $\alpha$, resultando em uma elipse a qual pode ser facilmente identificada e estabelecida. Comparações com os métodos clássicos de análise da estabilidade como o lugar das raízes, o conceito de entrada limitada-saída limitada, ou a estabilidade por Liapunov, também estão inclusas na análise do trabalho. Tais comparações têm indicado que os resultados obtidos através da metodologia proposta são menos conservativos e consequentemente produz uma análise e mapeamento da região de estabilidade robusta, considerando todas as variáveis, os parâmetros e as interações simultâneas entre eles, sem a necessidade de avaliar contribuições isoladas relativas a análise de estabilidade. O método proposto permite também monitorar continuamente a região de estabilidade, avaliando a sua tendência, e com isso determinado a necessidade de tomada de decisão, em tempo hábil, a fim de restaurar tal região.
\end{abstract}

\section{INTRODUÇÃO}

A estabilidade é uma das características mais importantes do sistema (Ogata, 2010). A análise da estabilidade deve ser aplicada não somente em relação a dinâmica do sistema de controle, mas também com relação as etapas iniciais dos projetos de tais sistemas. De acordo com Nerendra e Annaswamy (2012) o primeiro passo para verificar a estabilidade de um sistema seria o desenvolvimento de modelos do processo adequados a descrever o comportamento do sistema dinâmico.

Os trabalhos acadêmicos no âmbito de sistemas de controle têm tratado as incertezas, em sua maioria, como determinísticas ou vinculadas à estimação de probabilidade associada aos métodos clássicos de otimização. Nesse sentido, as perturbações introduzidas a um sistema estão associadas as raízes da equação característica, que são consideradas variáveis aleatória, havendo, portanto, uma distribuição de probabilidade vinculada a cada uma delas. 


\section{9 a 22 de outubro de 2014 \\ Florianópolis/SC}

Este trabalho apresenta os conceitos de estabilidade clássica com uma visão determinística, com o correspondente tratamento matemático, o qual não considera as efetivas interações entre as variáveis analisadas, como também a estabilidade do ponto de vista estatístico, com a qual se pode estabelecer a estabilidade do sistema para qualquer perturbação ocorrida no mesmo. Vale salientar o tratamento estatístico diz respeito ao universo de todos os resultados possíveis para o fenômeno. Diferente de outros métodos de análise da estabilidade, que requerem a estimação de probabilidade em sua essência, a estabilidade baseada em princípios estatísticos é direcionada a estabelecer a região de confiança para cada raiz da equação característica do processo (Silva et, al, 2012a).

Para identificar a condição de estabilidade do sistema, inclusive sob o aspecto dinâmico, este trabalho visa estabelecer o mapeamento do lugar onde se encontram as raízes no plano complexo determinando assim a região de estabilidade do sistema, além de estabelecer um comparativo entre as metodologias clássica e estatística.

\section{CONCEITO DE ESTABILIDADE CLÁSSICA}

A análise de estabilidade de um sistema tem sido normalmente realizada por duas teorias clássicas para equacionar os problemas derivados de tal análise: a teoria da estabilidade devida a Lyapunov e a teoria da estabilidade Entrada-Saída, as quais são baseadas em técnicas de análise funcional (Nerendra e Annaswamy 2012). Decorrentes de tais teorias surgem os conceitos para estabilidade de Lyapunov, que considera a estabilidade como uma propriedade interna do sistema e trata com o efeito de perturbações momentâneas, resultando em mudanças nas condições iniciais. Já as definições para a teoria da estabilidade entrada-saída, como o nome sugere, considera o efeito de pertubações externas sobre o sistema.

A definição de limites previamente estabelecidos para as variáveis de interesse, dentro dos quais o sistema deve permanecer estável, também tem sido adotado como uma estratégia para se garantir a estabilidade do sistema dinâmico. Entretanto, tais estatégias têm se mostrado substancialmente conservadoras.

\subsection{ESTABILIDADE E O LOCAL DAS RAÍZES}

As definições de estabilidade estão bem estabelecidas e exploradas não só do ponto de vista acadêmico, mas também como resultado da implementação prática

Assim, a definição de estabilidade para sistemas lineares pode ser resumida com a seguinte declaração: um sistema de controle linear é instável se qualquer das raízes de sua equação característica estiver sobre o eixo imaginário ou a sua direita. Caso contrário, o sistema é estável (Coughanowr e Koppel 1965).

Um dos problema da metodologia determinista reside em não considerar as interações entre as variáveis como também o assim chamado "problema inverso", podendo-se ainda observar que as raízes do sistema estão claramente associadas ao modelo probabilístico com densidade de probabilidade uniforme, esta mostrada na Figura 01. Desta forma, as raízes da equação característica podem ser estabelecidas com definição de sua localização. 


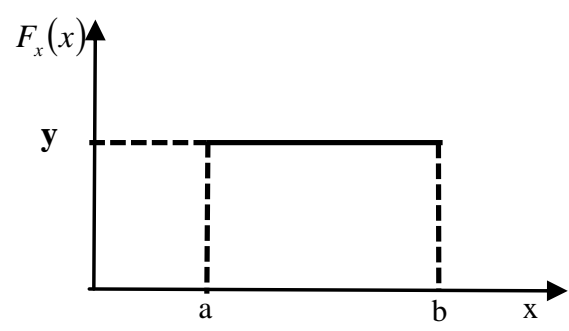

Figura 01 - Diagrama da distribuição uniforme.

\subsection{ESTABILIDADE DE LYAPUNOV}

Estabilidade para sistemas não-lineares dada pela teoria de Lyapunov. Consideremos o seguinte sistema:

$$
\dot{X}=A x \quad x\left(t_{0}\right)=x_{0}
$$

A técnica para determinar o comportamento das trajetórias da equação 01 nas vizinhanças da origem quando $\mathrm{F}(0)=0$, foi estudada pelo matemático russo A. A. Lyapunov e tem sido mencionado como sendo o método direto de Lyapunov, com mínimo local. Uma versão mais geral do lema Lyapunov tem sido usada, parcialmente, para mostrar a conexão entre estabilidade exponencial e observabilidade para sistemas lineares (Sastry e Bodson, 2011).

Teorema 01. Lyapunov:

(a) todos os autovalores A rementem no semi plano esquerdo aberto;

(b) $\mathrm{x}=0$ é um ponto de equilíbrio estável exponencialmente da equação 01 ;

(c) para todos os $\mathrm{C} \in \mathrm{R}^{\mathrm{m} \times \mathrm{n}}$ (com $\mathrm{m}$ arbitrário), tal que o par [C, A] é observável, existe uma simétrica positiva definida $\mathrm{P} \in \mathrm{R} .^{\mathrm{n} \times \mathrm{n}}$ que satisfaz;

$$
\mathrm{A}^{\mathrm{T}} \mathrm{P}+\mathrm{PA}=-\mathrm{C}^{\mathrm{T}} \mathrm{C}
$$

(d) para alguns $\mathrm{C} \in \mathrm{R}^{\mathrm{m} \times \mathrm{n}}$ (com m arbitrário), tal que o par [C, A] está sendo observado, existe uma simétrica positiva definida $\mathrm{P} \in \mathrm{R}^{\mathrm{n} \times \mathrm{n}}$ satisfazendo a Equação 02.

\subsection{ESTABILIDADE ENTRADA-SAIDA}

Outra abordagem geral da estabilidade é baseada nas técnicas de análise funcional, usada por Popov e posteriormente desenvolvida por Sandberg e Zames (Nerendra e Annaswamy, 2012). Os resultados obtidos com esse método de entrada e saída, apresentam uma grande semelhança aos resultados obtidos pelo método de Lyapunov.

A Figura 02 mostra um diagrama de um sistema por realimentação na sua forma canônica, a partir do qual uma análise mais detalhada da configuração resulta nas expressões descritas abaixo. Tal formalismo mostra-se particularmente útil na análise de estabilidade de sistemas interconectados, apresentando o problema típico do sistema determinístico por realimentação. 


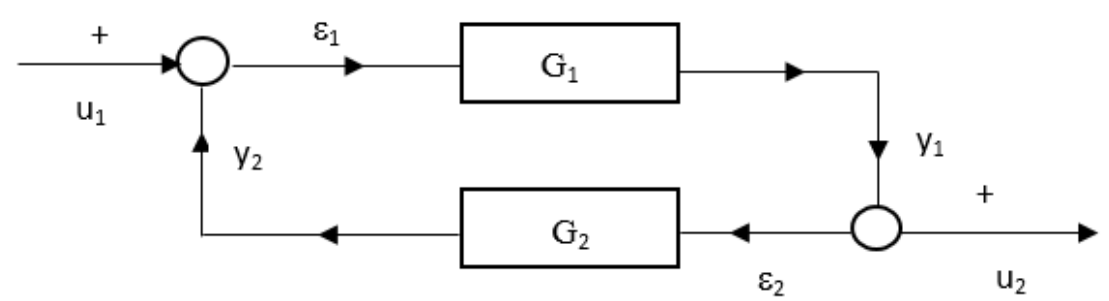

Figura 02 - um sistema canônico realimentado

Decorrente da Figura 02, as seguintes informações podem ser obtidas, resultando em:

$$
y_{1}=G_{1} \varepsilon_{1}(03) ; y_{2}=G_{2} \varepsilon_{2} \quad(04) ; \varepsilon_{1}=u_{1}-y_{2} \quad(05) ; \varepsilon_{2}=u_{2}-y_{1} \quad \text { (06). }
$$

com isso, as seguintes expressões para $y_{1}, y_{2}, \varepsilon_{1}$ e $\varepsilon_{2}$ são dadas por:

$$
\left\|y_{1}\right\| \leq \gamma_{1} \varepsilon_{1}+B_{1} \quad \text { (07); } \quad\left\|y_{2}\right\| \geq \gamma_{2} \varepsilon_{2}+B_{2} \quad(08) ; \quad\left\|\varepsilon_{1}\right\|=\left\|u_{1}-y_{2}\right\| \leq\left\|u_{1}\right\|+\left\|y_{2}\right\| \quad \text { (09). }
$$

Substituindo o valor de $\varepsilon_{2}$ na Equação 10, encontramos as seguintes correlações:

$$
\begin{aligned}
\left\|\varepsilon_{1}\right\| \leq\left\|u_{1}\right\|+\gamma_{2}\left\|\varepsilon_{2}\right\|+B_{1}(10) & \Rightarrow\left\|\varepsilon_{1}\right\| \leq\left\|u_{1}\right\|+\gamma_{2}\left(\left\|u_{2}\right\|-\left\|y_{1}\right\|\right)+B_{1} \\
& \Rightarrow\left\|\varepsilon_{1}\right\| \leq\left\|u_{1}\right\|+\gamma_{2}\left(\left\|u_{2}\right\|-\left\|\gamma_{1} \varepsilon_{2}+B_{2}\right\|\right)+B_{1}
\end{aligned}
$$

onde :

$$
\begin{aligned}
& \left\|\varepsilon_{1}\right\| \leq\left\|u_{1}\right\|+\gamma_{2}\left(\left\|u_{2}\right\|-\left\|\gamma_{1} \varepsilon_{2}+B_{2}\right\|\right)+B_{1} \quad \text { (13) } \Rightarrow\left\|\varepsilon_{1}\right\| \leq\left\|u_{1}\right\|+\gamma_{2}\left\|u_{2}\right\|-\gamma_{2} \gamma_{1}\left\|\varepsilon_{1}\right\|+\gamma_{2} B_{2}+B_{1} \\
& \Rightarrow\left(1-\gamma_{2} \gamma_{1}\right)\left\|\varepsilon_{1}\right\| \leq\left\|u_{1}\right\|+\gamma_{2}\left\|u_{2}\right\|+\gamma_{2} B_{2}+B_{1} \quad \text { (15) } \Rightarrow\left\|\varepsilon_{1}\right\| \leq \frac{1}{\left(1-\gamma_{2} \gamma_{1}\right)}\left[\left\|u_{1}\right\|+\gamma_{2}\left\|u_{2}\right\|+\gamma_{2} B_{2}+B_{1}\right]
\end{aligned}
$$

Portanto, considerar $\gamma_{1} \gamma_{2} \cong 1$ implica em uma região instável para o sistema realimentado, desde que a Equação 16 tende a infinito. A estabilidade só será mantida caso $\gamma_{1} \gamma_{2} \neq 1$. Vale ressaltar que tal resultado é alcançado para os casos onde nenhuma correlação entre as variáveis é verificada.

\section{ASSOCIAÇÃO ENTRE ESTABILIDADE ROBUSTA DETERMINÍSTICA E A ESTABILIDADE SOBRE A ÓTICA ESTATÍSTICA}

A estabilidade robusta determinística pode ser considerada associada ao conceito de distribuição uniforme, cuja variáveis aleatórias são uniformemente distribuída como uma função densidade de probabilidade, que é constante sobre o intervalo em consideração. Na análise da estabilidade do ponto de vista determinístico para sistemas de várias variáveis, estabelece-se a faixa de variação de cada variável pelo cruzamento dos limites extremos de cada variável e para tal especificação são determinadas as condições do processo para se manter estável, ou seja, a região de estabilidade robusta seria para duas variáveis, por exemplo, dada pelo retângulo da Figura 03(a). Também pode ser observado que esta figura reflete o mesmo comportamento apresentado pela distribuição uniforme bivariada, ou seja, dizer que as variáveis não tem grau algum de interação pode ser semelhante dizer que obedecem a uma distribuição uniforme de 
acordo com a Figura 3(b) do ponto de vista estatístico, visto que não há contração ou descontração de espaços. Entretanto, não é razoável assumir que as variáveis aleatórias sejam sempre distribuídas uniformemente. Portanto, a análise do ponto de vista determinístico mostrase ser um caso particular da análise de estabilidade sob ótica estatística.

a)

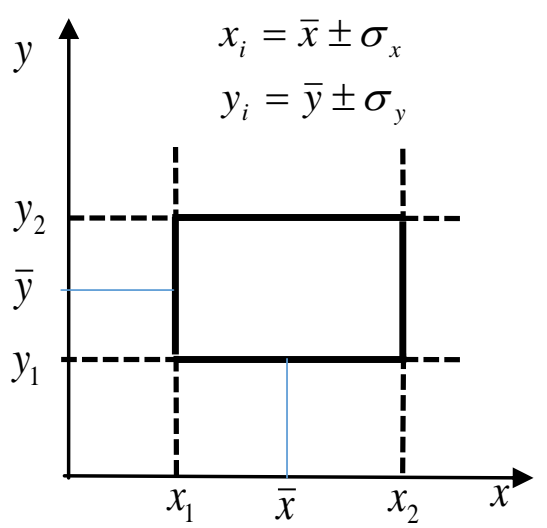

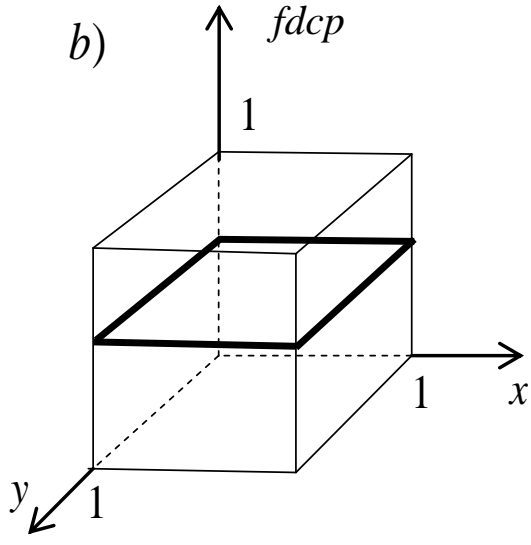

Figura 03 - a) Representação da região conjunta bivariada. b) Função de distribuição uniforme bivariada $(f d c p)$.

A Figura 03 (b) representa a função de distribuição conjunta de probabilidade ( $f d c p$ ), onde admiti-se a independência das variáveis. Mostra tambem a forma de uma superfície plana limitada pelos eixos x e y, cuja representação revela a correspondência com a Figura 3 (a).

\section{ESTABILIDADE BASEADA EM PRINCÍPIOS ESTATÍSTICOS}

De forma diferente de outros métodos que requerem a estimação de probabilidade em suas estratégias, a Estabilidade baseada em princípios estatísticos é direcionada a estabelecer a região de confiança para cada raiz da equação característica do processo em questão.

A técnica usada considera a relação existente entre a métrica Euclidiana e a distância estatística. A distância estatística tem essa denominação uma vez que utiliza variância e covariância das amostras. Para tornar possível a determinação dos contornos da elipse, região de confiança, as formas quadráticas são estabelecidas. Esses resultados se originam das partes reais e imaginarias de números complexos vinculados à distribuição chi-quadrado (Silva et. al, 2012b).

\subsection{FORMULAÇÃO MATEMÁTICA}

Para formular essa teoria, considera-se um ponto $\mathrm{P}$ qualquer com coordenadas $\mathrm{x}_{1}$ (parte real) e $\mathrm{x}_{2}$ (parte imaginaria) em um plano complexo onde a distância Euclidiana de $\mathrm{P}$ para origem $\mathrm{O}$ e dada pela Equação 17 (Silva et. al, 2014).

$$
d(O, P)=\sqrt{\left(x_{1}\right)^{2}+\left(x_{2}\right)^{2}}
$$


Normalizando as coordenadas obtemos a distância estatística:

$$
d(O, P)=\sqrt{\left(\frac{x_{1}}{\sigma_{x_{1}}}\right)^{2}+\left(\frac{x_{2}}{\sigma_{x_{2}}}\right)^{2}}
$$

Em notação vetorial pode ser escrita como sendo $d^{2}(O, P)=X^{\prime} \sum^{-1} X$ onde $\sigma_{x i}$ é e a variância de componentes do vetor $X$, daí $X^{\prime}=\left[x_{1}, x_{2}\right]$ e o somatório é dado por:

$$
\sum=\left[\begin{array}{cc}
\sigma_{x 1}^{2} & 0 \\
0 & \sigma_{x 2}^{2}
\end{array}\right]
$$

Generalizando a Equação 19, temos que:

$$
d^{2}(\mu, P)=\left(X-\mu_{x}\right)^{\prime} \sum^{-1}\left(X-\mu_{x}\right)
$$

Na qual descreve a equação de uma elipse com centro no valor esperado da variável aleatória X. Para uma variável bidimensional a Equação 20 descreve os contorno de uma elipse centrada em $\mu\left(\mu_{x 1}, \mu_{x 2}\right)$, onde os seus eixos podem não ser necessariamente na mesma direção que o plano de coordenadas.

Sem perder a generalidade, a Figura 05 ilustra o gráfico da Equação 20, conforme a Figura 05:

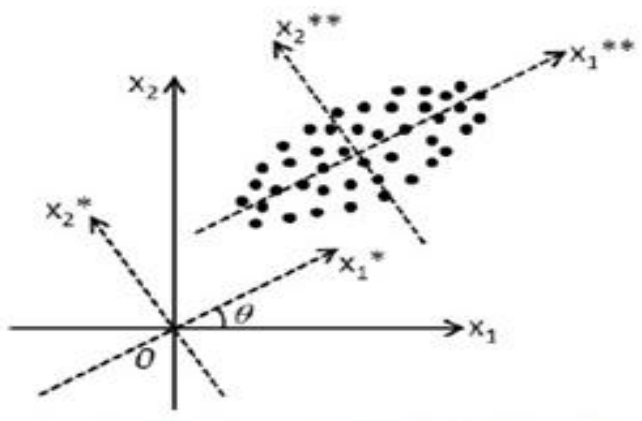

Figura 05 - Representação gráfica da equação 20.

O movimento de rotação e a translação dos eixos podem ser expressos pela Equação $(X-\overline{\mathrm{X}})=\mathrm{P}\left(X^{*}-\overline{\mathrm{X}}^{*}\right)$, resultando na supressão dos termos de covariância cruzada.

Onde a matriz ortogonal $P$ é dada por:

$$
P=\left[\begin{array}{cc}
\cos \theta & -\sin \theta \\
\sin \theta & \cos \theta
\end{array}\right]
$$

Para assegurar o movimento de rotação é necessário que o valor do determinante de $\mathrm{P}$ (det P) seja igual a 1 . Os eixos $\left(x_{1}^{*}, x_{2}^{*}\right)$ são rotacionados por um ângulo $\theta$ a partir de $\left(x_{1}, x_{2}\right)$. 
Substituindo a Equação 21 na Equação 20 obtemos a seguinte equação:

$$
d^{2}(\mu, P)=\left(X^{*}-\mu_{X^{*}}\right)^{\prime} P^{\prime} \sum{ }^{-1} P\left(X^{*}-\mu_{X^{*}}\right)
$$

Uma vez que a matriz $\Sigma^{-1}$ é simétrica e $\mathrm{P}$ uma matriz ortogonal, é possível diagonalizar $\Sigma^{-1}$ resultando em uma matriz diagonal $\mathrm{D}$, ou seja $D^{-1}=P^{\prime} \sum^{-1} P$, substituindo esta equação na Equação 24 obtemos a seguinte equação:

$$
d^{2}\left(\mu_{X^{*}}, P\right)=\left(X^{*}-\mu_{X^{*}}\right)^{\prime} D^{-1} \sum^{-1}\left(X^{*}-\mu_{X^{*}}\right)
$$

Vale salientar que a Equação 23 descreve a forma quadrática de uma elipse centrada no valor esperado da variável aleatória $X^{*}$ sem o termo de covariância cruzada.

Pode se reescrever a Equação 23 para duas dimensões, assim obtemos a Equação 24:

$$
d\left(\mu_{X^{*}}, P\right)^{2}=\frac{\left(x_{1}^{*}-\mu_{x_{1}^{*}}\right)^{2}}{\sigma_{x_{1}^{*}}^{2}}+\frac{\left(x_{2}^{*}-\mu_{x_{2}^{*}}\right)^{2}}{\sigma_{x_{2}^{*}}^{2}}
$$

Considerando a definição da variável $\chi^{2}$ (chi-quadrado) através da expressão matemática mostrada abaixo

$$
\chi^{2}=U_{1}^{2}+\ldots+U_{v}^{2}=\sum_{i=1}^{v} U_{i}^{2}=\sum_{i=1}^{v}\left(\frac{X_{i}-\mu_{i}}{\sigma_{i}}\right)^{2}
$$

observa-se que $X_{i}-\mu_{i}$ está relacionada com a média e $v$ é chamando de grau de liberdade. Das equações 23 e 25 verifica-se que $d\left(\mu_{X^{*}}, P\right)^{2}$ possui uma distribuição $\chi^{2}$, ou seja, pode ser representada por uma $\chi^{2}$ com $v$ grau de liberdade e como resultado obtemos a Equação 29. Vale salientar que o contorno de uma elipse é determinado como sendo $(1-\alpha) 100 \%$ da probabilidade para o qual $\alpha$ corresponde a um desejado nível de significância e $v=2$.

$$
d\left(\mu_{X^{*}}, P\right)^{2}=\frac{\left(x_{1}^{*}-\mu_{x_{1}^{*}}\right)^{2}}{\sigma_{x_{1}^{*}}^{2}}+\frac{\left(x_{2}^{*}-\mu_{x_{2}^{*}}\right)^{2}}{\sigma_{x_{2}^{*}}^{2}} \cong \chi^{2}
$$

O conjunto de pontos dado pela distância estatística circunscreve o contorno do elipsoide, o qual limita região da estabilidade para um determinado nível de significância $\alpha=0,05$, podendo então ser mapeado.

\section{CONCLUSÕES}

Uma metodologia foi desenvolvida tendo como objetivo estimar a região robusta de confiança para a estabilidade e, consequentemente, mapear os contornos das elipses resultantes associadas com as raízes aleatórias da equação característica. O procedimento adota o nível de significância $\alpha=0,05$. 


\section{9 a 22 de outubro de 2014 \\ Florianópolis/SC}

A metodologia estabelece os limites para as variáveis de interesse, raízes do sistema, com base nos princípios estatísticos, e com isso a região de confiança robusta. Tal metodologia implica uma associação do conceito de distância estatística com a distribuição qui-quadrado.

Uma vez associada a estabilidade robusta determinística à estabilidade sobre a ótica estatística, pode ser observado que a estabilidade robusta determinística segue o modelo da distribuição uniforme, onde a probabilidade das raízes características é a mesma durante todo intervalo, enquanto o modelo estatístico trabalha com a determinação da região de estabilidade robusta levando em consideração as interações entre todas as variáveis consideradas no processo. Isto tem permitido mapear a região de estabilidade com possibilidade de monitoramento online do sistema

\section{AGRADECIMENTOS}

O Conselho Nacional de Desenvolvimento Científico e Tecnológico (CNPq) pelo financiamento da pesquisa, e a Unidade Acadêmica de Engenharia Química da Universidade Federal de Campina Grande (UFCG)

\section{$7 \quad$ BIBLIOGRAFIA}

COUGHANOWR, Donald R.; KOPPEL, Lowell B. Process systems analysis and control. New York: McGraw-Hill, 1965.

NARENDRA, Kumpati S.; ANNASWAMY, Anuradha M. Stable adaptive systems. Courier Dover Publications, 2012.

OGATA, K. Engenharia de Controle Moderno, 5.ed. São Paulo: Prentice Hall 2010.

RIBEIRO, Ademir Alves; KARAS, Elizabeth Wegner. Otimização contínua Aspectos teóricos e computacionais. Cengage Learning, São Paulo, Brazil, in press (in Portuguese) 2013.

SASTRY, Shankar; BODSON, Mark. Adaptive control: stability, convergence, and robustness, 2011.

SILVA, N., BISPO, H., BRITO, R. and MANZI, J. (2014), Robust stability analysis inspired by classical statistical principles. Can. J. Chem. Eng., 92: 82-89. doi: 10.1002/cjce.21825

SILVA, J. N. Determinação da região robusta de estabilidade inspirada nos princípios da estatística clássica Campina Grande 2013.

SILVA, J. N. ; BISPO, H. J. ; BRITO, R. P. ; MANZI, J. T. . Analise estatística aplicada a estabilidade robusta. In: XIX Congresso Brasileiro de Automática, CBA 2012, 2012, Campina Grande. Anais do XIX Congresso Brasileiro de Automática, CBA 2012, $2012 \mathrm{a}$.

SILVA. J. N BISPO. H. BRITO R. MANZI. J. Joint confidence region for tuning parametres of the PID controller. In IFAC Conference on Advanced in PID control, 2012 Brescia. IFAC PID'12 - Book of abstract Brescia University of Brescia 2012b v1

SOONG, T. T. Modelos probabilísticos em engenharia e ciências. LTC, 1986. 